\title{
Determinación de microorganismos fúngicos en semillas de Amaranto (Amaranthus spp.) mediante diferentes métodos de análisis
}

\section{Determination of fungi microorganisms on Amaranto seeds (Amaranthus spp.) by different analysis methods}

\author{
Maria C. Noelting ${ }^{1}$, Maria C. Sandoval ${ }^{2}$, Nilda N. Abbiati ${ }^{2}$
}

Presentado: $07 / 09 / 2004$

Aceptado: $\quad 08 / 11 / 2004$

\section{Resumen}

La ausencia de una metodología destinada al análisis de la micoflora en semillas de amaranto (Amaranthus spp.) ha motivado la realización del presente trabajo. A tal efecto, fueron sembradas semillas de dos cultivares (con y sin tratamiento de desinfección en forma previa a la siembra) en papel «Blotter test» y en cinco medios agarizados: Agar Papa Glucosado al 2\% (APG), Agar Extracto de Glucosa Cloramfenicol (CYG), Agar Czapek (CZ), Agar para conteo en placa (PCA) y Agar Sabouraud (SAB). Los cajas fueron incubadas a $26^{\circ} \mathrm{C} \pm 2$ y $16 \mathrm{hs}$ luz/8hs oscuridad de fotoperíodo durante siete días, a partir del cual se procedió a registrar el número máximo de géneros fúngicos desarrollados y el porcentaje de germinación de las semillas. Un total de catorce géneros fúngicos procedentes del campo y del almacenamiento fueron registrados, destacándose Alternaria por su mayor frecuencia de aislamiento. Al evaluar el número máximo de géneros fúngicos, se detectaron diferencias significativas entre los medios $(p>0,001)$ resultando los medios APG, CZ y PCA los mas efectivos. Por otro lado, la desinfección aplicada a las semillas redujo en términos generales el desarrollo de hongos de crecimiento expansivo. El análisis de los datos correspondientes al porcentaje de germinación reveló la presencia de interacciones entre los cultivares y los niveles de desinfección significativas $(p>0,001)$ en cuatro de los medios analizados; mientras que en el resto se registraron interacciones no significativas. test

Palabras clave: amaranto, Amaranthus, fitopatología, Alternaria, semillas, medios agarizados, blotter

\section{Abstract}

The absence of a methodology to analyze the micoflora in amaranth's seed, has motivated the performing of the present work. Seeds of two cultivars were sown (with and without disinfection process before sowing) on Petri dishes, some with "Blotter test" paper and others with five agarized culture media. All of them were placed in a chamber with $26^{\circ} \mathrm{C} \pm 2$ and $16 \mathrm{~h}$ of photoperiod, and were incubated during seven days. After this period of time, the maximum number of different fungi genera developed on each substrate was registered, and the seed germination percentage was calculated. A total of fourteen genera of fungi (field and storage ones) were found, being Alternaria the most frequently isolated. In relation to the maximum number of fungi genera found, significant differences among the media analyzed $(p>0,001)$ were detected, resulting the media APG, CZ and PCA the most effective ones. According to the disinfection treatment applied to the seeds before sowing, in general terms the fungi expansive growth was reduced. The germination percentage analysis revealed the presence of significant $(p>0,001)$ interactions between amaranth cultivars and disinfection levels in four of the media analyzed, while in the remainder not significant interactions were registered.

keywords: amaranth, Amaranthus, Phytopathology, Alternaria, seeds, agarized media, blotter test.

${ }^{1}$ IFSC, CIGEN Facultad de Ciencias Agrarias y Forestales. Universidad Nacional de La Plata, Garibaldi 3400 CP(1836) Llavallol. Buenos Aires - Argentina.

Email: María C. Noelting mcnoelting@ hotmail.com

${ }^{2}$ Facultad de Ciencias Agrarias. Universidad Nacional de Lomas de Zamora Ruta 4 Km 2 Llavallol, Provincia de Buenos Aires - Argentina 


\section{Introducción}

La importancia del cultivo de amaranto ha sido reconocida entre otros ámbitos científicos por la Academia Nacional de Ciencias de los Estados Unidos, considerando como relevante su promisorio valor económico (National Academy of Sciences, 1975).

Se trata de un cultivo apto para la alimentación humana y animal. Así como también abono verde, combustible y con múltiples aplicaciones en la industria textil y farmacéutica entre otras.

Una de las razones del creciente interés que ha despertado en los últimos años en investigadores de varios países es la calidad nutricional de sus semillas, las que poseen un porcentaje de proteínas que varía del $12,5 \%$ al $17,6 \%$; además destaca su alto nivel de lisina que alcanza el $5 \%$, característica que las diferencia de otros cereales y pseudocereales (Afolabi et al., 1981; Becker et al., 1981; Carlsson, 1980; Schmidt, 1977; Uzo and Okorie, 1983).

En Argentina, el cultivo de amaranto se considera como una producción con alto potencial y de interés estratégico. Ello se debe a las cualidades nutricionales y a la capacidad que tiene el cultivo de adaptarse a diversas condiciones hídricas y edáficas. Estas últimas características permitirán el establecimiento de dicho cultivo en un área equivalente a cinco millones de hectáreas (Covas, 1990).

Actualmente no se dispone oficialmente de un método de análisis que permita evaluar el estado sanitario de las semillas de amaranto. Las referencias sobre este tema en particular son escasas. Cabe mencionar el trabajo realizado por Bartolini \& Hamptom (1989), quienes han determinado el grado de contaminación fúngica de las semillas de amaranto de acuerdo a su posición en las inflorescencias.

El objetivo el presente trabajo ha sido evaluar métodos de análisis sanitarios mas adecuados para aislar microorganismos fúngicos presentes en semillas de amaranto y determinar la influencia de la desinfección de las semillas y de los métodos de análisis utilizados en su germinación.

\section{Material y Métodos}

Se emplearon un total de 3600 semillas correspondientes a dos cultivares de amaranto: Amaranthus cruentus cv. Don Guiem y $A$. hyponchondriacus cv G. Covas, ambos procedentes de la Estación Experimental Instituto Nacional de Tecnología Agropecuaria Inta Anguil, ubicada en la provincia de La Pampa, Argentina. Las semillas no recibieron tratamientos químicos ni físicos tendientes a inducir la germinación.

Para evaluar la micoflora presente en las semillas, se procedió a sembrar las mismas en papel de filtro absorbente Whatman $\mathrm{N}^{\circ} 2$ (Blotter test) y en cinco medios agarizados: Agar Papa Glucosado al 2\% (APG), Agar Extracto de Glucosa Cloramfenicol (CYG), Agar Czapek (CZ), Agar para conteo en placa (PCA) y Agar Sabouraud (SAB) contenidos en cajas de Petri respectivamente. Todos los medios agarizados fueron ajustados a un mismo $\mathrm{pH}(6,5)$.

Previa a su siembra, las semillas fueron desinfectadas con una solución de hipoclorito de sodio al $2 \%$, durante 5 minutos, posteriormente enjuagadas tres veces con agua destilada estéril y secadas entre papeles de filtro estériles. Las semillas fueron dispuestas en grupos de cincuenta por cada caja de Petri de 9 $\mathrm{cm}$ de diámetro. Los testigos consistieron en la siembra de las semillas sin desinfectar en los substratos anteriormente citados.

Las cajas una vez sembradas fueron dispuestas en una cámara a $26^{\circ} \mathrm{C} \pm 2$ y $16 \mathrm{hs}$ luz/8 hs oscuridad de fotoperíodo durante siete días. Luego del período de incubación se procedió a identificar los microorganismos fúngicos presentes sobre la base de la observación de caracteres macro-microscópicos y el uso de claves apropiadas (Barnett \& Hunter,1998; Nelson et al., 1983; Pitt \& Hocking, 1985). 
Se cuantificó el número de géneros fúngicos desarrollados y el porcentaje de germinación de las semillas. El criterio para evaluar la efectividad de los métodos de análisis sanitarios se basó en el número de géneros fúngicos desarrollados a partir de las semillas presentes por cada unidad experimental, en este caso por cada caja de Petri.

Se llevaron a cabo análisis complementarios con la finalidad de determinar el carácter patógeno de los microorganismos fúngicos aislados. Para realizar las pruebas de patogenicidad se tomó como criterio la elección de aquellos microorganismos aislados con mayor frecuencia tanto de semillas como de plantas sintomáticas cultivadas en el campo.

La identificación a nivel específico fue realizada para aquellos microorganismos de patogenicidad comprobada. Para efectuar las pruebas de patogenicidad se emplearon cultivos de ambos patógenos desarrollados en APG durante siete días bajo condiciones de $26^{\circ} \mathrm{C}$ y $16 \mathrm{~h}$ de fotoperiodo. A partir de colonias desarrolladas se obtuvieron con sacabocados secciones de las mismas de $0,9 \mathrm{~mm}$ de diámetro con las que se inocularon plantas y semillas de ambos cultivares de amaranto. La evaluación del estado sanitario de plantas de ambos cultivares de amaranto fue realizada en un lote experimental.

El material vegetal procedente de plantas afectadas fue desinfectado y sembrado en cajas con APG. Los microorganismos que desarrollaron fueron identificados procediéndose luego a su aislamiento y cultivo.

\section{Análisis estadístico}

El ensayo fue realizado por triplicado según un diseño completamente aleatorizado en arreglo factorial con una combinación de 24 tratamientos que incluyeron a dos cultivares de amaranto, dos niveles de desinfección correspondientes a semillas sin desinfectar y desinfectadas y seis métodos de análisis sanitarios que incluyeron al «Blotter test»y a cinco medios agarizados: APG, CZ, PCA, CYG, SAB.
Los datos correspondientes al número de géneros fúngicos presentes en semillas por cada caja de Petri y al porcentaje de germinación de las semillas fueron sometidos a una transformación previa al análisis de varianza. Se utilizaron las transformaciones raíz cuadrada y arcoseno para la primera y segunda variable respuesta.

El análisis fue complementado con la aplicación de contrastes ortogonales y pruebas de comparaciones múltiples de Tukey con un nivel de significación del 5\% mediante el programa Statystical Análisis System (SAS) (1989).

\section{Resultados}

\section{Géneros fúngicos presentes en semillas de amaranto}

Se registraron un total de catorce géneros fúngicos (de campo y de almacenamiento) siendo Alternaria el género fúngico aislado con mayor frecuencia. Las semillas de $A$. hypochondriacus exhibieron una mayor contaminación por Mucor, Rhizopus, Fusarium; en tanto que las semillas de A.cruentus presentaron una mayor contaminación por Alternaria, Aspergillus y Penicillium (Tabla 1).

La evaluación de la efectividad de los métodos de análisis sanitarios demostró que el número de géneros fúngicos aislados de las semillas de amaranto, estuvo condicionado por los métodos de análisis empleados, independientemente de los cultivares de amaranto analizados y de los niveles de desinfección considerados.

Todos los medios agarizados (APG, CYG, PCA, CZ y SAB) se diferenciaron del «Blotter test» (b-test) en cuanto al número de géneros fúngicos aislados, resultando los medios $\mathrm{CZ}$, APG y PCA los más efectivos para recuperar los microorganismos presentes en las semillas de amaranto (Tabla 1 y 2).

Con respecto a la desinfección aplicada a las semillas, se registraron cambios en cuanto al tipo y frecuencia de géneros fúngicos aislados. Estos cambios han implicado: 
Tabla 1. Hongos aislados de semillas de dos especies de Amaranthus. Los datos que figuran entre paréntesis corresponden a la frecuencia de aparición de cada género fúngico en particular.

\begin{tabular}{|c|c|c|c|c|c|}
\hline \multicolumn{2}{|c|}{ Métodos de } & \multicolumn{2}{|c|}{ A. cruentus cv D on G uiem } & \multicolumn{2}{|c|}{ A. hypochondriacus cv G Covas } \\
\hline análisis & Hongos & Sin desinfectar & D esinfectadas & Sin desinfectar & D esinfectadas \\
\hline \multicolumn{6}{|l|}{ APG } \\
\hline & A cremonum & -- & $(12,5 \%)$ & -- & - \\
\hline & A lternaria & $(41,66 \%)$ & $(25,0 \%)$ & -— & $(12,5 \%)$ \\
\hline & A spergillus & $(12,5 \%)$ & -- & -— & -- \\
\hline & Arthrinium & - & $(12,5 \%)$ & -- & - - \\
\hline & A ureobasidium & -— & $(12,5 \%)$ & - - & - - \\
\hline & Colletotrichum & - - & - & - - & $(6,25 \%)$ \\
\hline & Crysosporium & -- & $(12,5 \%)$ & -- & -- \\
\hline & Fusarium & $(8,33 \%)$ & - - & -— & $(25 \%)$ \\
\hline & elminthosporium & $(12,5 \%)$ & -- & - - & - - \\
\hline & M ucor & $(8,33 \%)$ & - - & $(75 \%)$ & - - \\
\hline & Penicillium & $(8,33 \%)$ & - - & -- & -- \\
\hline & Levaduras & - & $(25,0 \%)$ & -- & $(12,5 \%)$ \\
\hline & Rhizopus & -- & -- & (25\%) & - - \\
\hline \multicolumn{6}{|l|}{ CYG } \\
\hline & Alternaria & $(25 \%)$ & $(8,33 \%)$ & $12,5 \%)$ & -— \\
\hline & A spergillus & $(25 \%)$ & - - & -- & - - \\
\hline & Fusarium & -- & - - & -- & $(33,33 \%)$ \\
\hline & elminthosporium & $(25 \%)$ & $(25,0 \%)$ & - - & -- \\
\hline & M ucor & - & - - & $(25 \%)$ & - - \\
\hline & Rhizopus & - - & - - & (25\%) & - - \\
\hline & Rhizoctonia & -- & - - & -- & $(25 \%)$ \\
\hline \multicolumn{6}{|l|}{ PCA } \\
\hline & Alternaria & $(12,5 \%)$ & $(20,83 \%)$ & $(41,66 \%)$ & $(25 \%)$ \\
\hline & Fusarium & -- & -- & $(8,33 \%)$ & $(61,5 \%)$ \\
\hline & Levaduras & $(24,99 \%)$ & $(45,83 \%)$ & $(8,33 \%)$ & $(12,5 \%)$ \\
\hline & M ucor & -— & - - & $(20,83 \%)$ & - - \\
\hline & Penicillium & $(16,66 \%)$ & -- & -- & - - \\
\hline & Stemphylium & $(20,84 \%)$ & $(8,33 \%)$ & $(20,83 \%)$ & - - \\
\hline \multicolumn{6}{|l|}{$C Z$} \\
\hline & Alternaria & $(37,49 \%)$ & $(58,33 \%)$ & $(41,66 \%)$ & (25\%) \\
\hline & Fusarium & -- & -- & $(20,83)$ & $(62,5 \%)$ \\
\hline & Levaduras & $(24,99 \%)$ & $(25,0 \%)$ & $(8,33 \%)$ & $(12,5 \%)$ \\
\hline & M ucor & -- & -- & $(8,33 \%)$ & -- \\
\hline & Penicillium & $(16,66 \%)$ & -— & -- & -— \\
\hline & Rhizopus & -- & -- & $(20,83 \%)$ & - - \\
\hline & Stemphylium & $(20,83 \%)$ & $(8,33 \%)$ & -- & -- \\
\hline \multicolumn{6}{|l|}{ SAB } \\
\hline & Alternaria & $(12,5 \%)$ & $(25,0 \%)$ & - & -— \\
\hline & A spergillus & $(12,5 \%)$ & -- & $(12,5 \%)$ & -- \\
\hline & Levaduras & $(37,5 \%)$ & $(25,0 \%)$ & $(37,5 \%)$ & (75\%) \\
\hline & Rhizoctonia & -- & -- & $(12,5 \%)$ & -- \\
\hline \multicolumn{6}{|l|}{ Blotter test } \\
\hline & Alternaria & -- & -- & (25\%) & -- \\
\hline & Rhizopus & -- & -- & (25\%) & -- \\
\hline
\end{tabular}

i) La eliminación de hongos de almacenamiento principalmente y la reducción en general de los hongos de campo. En términos generales la desinfección aplicada a las semillas inhibió el desarrollo de hongos de crecimiento expansivo tales como: Aspergillus, Rhizopus y Penicillium que tienen la habilidad de cubrir rápidamente los medios impidiendo la manifestación de otros microorganismos de crecimiento más lento. Como ejemplos se pueden citar a semillas de $A$. cruentus sembradas en los medios APG y CZ y a semillas de $A$. hypochondriacus sembradas en los medios $\mathrm{CZ}$ y $\mathrm{SAB}$ respectivamente.

ii) La manifestación de hongos presumiblemente presentes en las semillas en estado latente. Como ejemplo podemos citar 
Tabla 2. Número de géneros fúngicos presentes en semillas de Amaranthus spp. Prueba de Rango de Tukey (HSD). Prueba para la variable $R$ Nugen $\alpha=0.0572 \mathrm{gl}$. Valor crítico de rango studentizado 4,14. Diferencia Mínima significativa 0,2665 . Las medias seguidas por una letra en común dentro de una misma columna no difieren estadísticamente según el test de Tukey $(p=0,05)$.

la presencia en el medio APG de Acremonium, Arthrinium, Aureobasidium, Crysosporium, Fusarium, Colletotrichum y Rhizoctonia en semillas desinfectadas de A. hypochondriacus.

iii) El incremento en las semillas de ciertos géneros fúngicos de campo como consecuencia de la reducción de hongos de crecimiento expansivo. Esta situación se presentó en los medios PCA, CZ y SAB donde los porcentajes de Alternaria se incrementaron en las semillas desinfectadas de $A$. cruentus. Lo mismo ocurrió con Fusarium, cuya presencia se

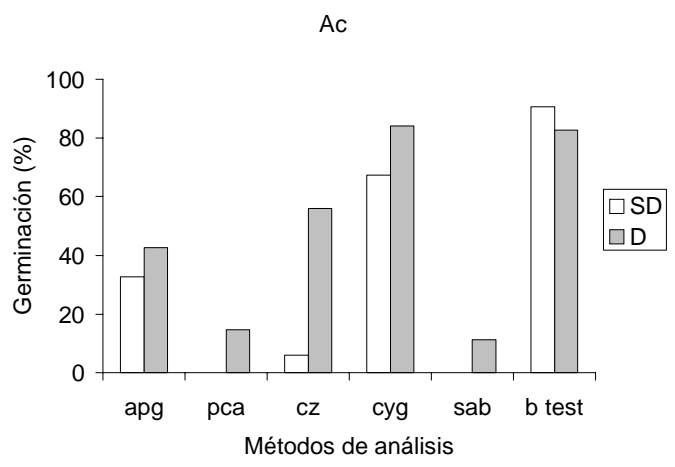

\begin{tabular}{lcc}
\hline $\begin{array}{l}\text { Métodos de } \\
\text { análisis }\end{array}$ & $\begin{array}{c}\text { Número promedio degéneros } \\
\text { fúngicos aislados } \\
\text { Valores } \\
\text { originales }\end{array}$ & $\begin{array}{c}\text { Valores } \\
\text { transformados }\end{array}$ \\
\hline CZ & 2 & $1,70 \mathrm{a}$ \\
APG & 1,62 & $1,58 \mathrm{ab}$ \\
PCA & 1,5 & $1,57 \mathrm{ab}$ \\
CYG & 1,06 & $1,40 \mathrm{~b}$ \\
SAB & 1 & $1,39 \mathrm{~b}$ \\
Blotter Test & 0,18 & $1,07 \mathrm{c}$ \\
\hline
\end{tabular}

incrementó en semillas desinfectadas de $A$. hypochondriacus desarrolladas en los medios PCA y CZ.

iv) Todos los métodos de análisis exceptuando el medio CYG y el Blotter test han sido efectivos para aislar levaduras de las semillas de amaranto.

\section{Porcentaje de germinación}

Los valores correspondientes al porcentaje de germinación debieron ser transformados para poder analizarlos estadísticamente (Tabla 3).

El análisis determinó una interacción significativa entre los cultivares, métodos analizados y niveles de desinfección aplicados, por dicho motivo se realizó un análisis de varianza para cada método en particular. Como resultado de dichos análisis, se registraron interacciones entre variedades de amaranto y niveles de desinfección significativas y no significativas.

Las interacciones significativas se han manifestado en los medios APG, CZ, CYG y PCA reportándose a continuación una información mas detallada:

i) En los medios CYG y APG (Tabla $3 b-c$ y Fig. 2b-c) si bien la germinación de los cultivares de amaranto se ha incrementado con la desinfección aplicada a sus semillas, la magnitud de la germinación no ha sido similar para

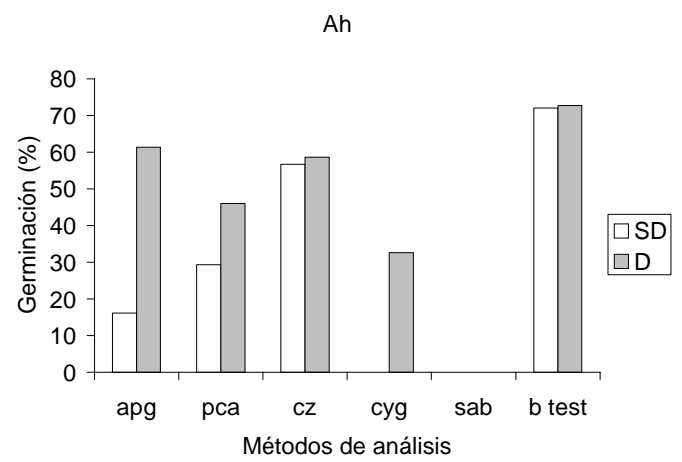

Figura 1. Porcentajes de germinación de amaranto según métodos de análisis y niveles de desinfección. (Ac) A. cruentus, (Ah) A. hypochondriacus. SD y D corresponden a semillas sin desinfectar y desinfectadas. Los valores de germinación corresponden a los porcentajes originales. 
Tabla 3. Germinación está expresada como el arcoseno de la raíz cuadrada de la proporción de la germinación mas 0,01 . $\mathrm{A} h=A$. hypochondriacus y $\mathrm{Ac}=A$.cruentus. $\mathrm{D}$ y $\mathrm{SD}$ : Con y $\sin$ Desinfección. SD Desvío estandar. Las medias seguidas por una letra en común dentro de la misma columna no difieren estadísticamente según el test de Tukey al $5 \%$ de probabilidades.

\begin{tabular}{|c|c|c|c|}
\hline $\begin{array}{l}\text { a) medio CZ } \\
\text { Desinfección }\end{array}$ & Cultivar & G erminación & DS \\
\hline $2-C D$ & 2-Ah & $0,88 a$ & 0,14 \\
\hline 1-SD & 2-Ah & $0,86 a$ & 0,03 \\
\hline $2-C D$ & $1-A C$ & $0,85 a$ & 0,05 \\
\hline 1-SD & $1-A C$ & $0,26 b$ & 0,04 \\
\hline $\begin{array}{l}\text { c) medio A PG } \\
\text { Desinfección }\end{array}$ & Cultivar & G erminación & DS \\
\hline $2-C D$ & 2-Ah & $0,91 a$ & 0,08 \\
\hline 2-CD & $1-A c$ & $0,72 b$ & 0,03 \\
\hline 1-SD & $1-A c$ & $0,61 b$ & 0,03 \\
\hline 1-SD & 2-Ah & $0,41 \mathrm{C}$ & 0,09 \\
\hline $\begin{array}{l}\text { e) medio PCA } \\
\text { Desinfección }\end{array}$ & Cultivar & G erminación & DS \\
\hline $2-C D$ & $2-A h$ & $0,75 a$ & 0,04 \\
\hline 1-SD & 2-Ah & $0,58 b$ & 0,06 \\
\hline 2-CD & $1-A C$ & $0,40 c$ & 0,03 \\
\hline 1-SD & $1-A C$ & $0,10 \mathrm{~d}$ & 0,10 \\
\hline
\end{tabular}

ambos cultivares. En este caso los incrementos registrados en A. hypochondriacus fueron superiores a los registrados en A. cruentus.

ii) En los medios CZ y SAB (Tabla 3a-d y Fig. 2a-d) se han registrado incrementos significativos en la germinación de semillas desinfectadas de A. cruentus. En cambio, en las semillas de A. hypochondriacus no se registraron variaciones en su germinación considerando los dos niveles de desinfección.

Las interacciones no significativas se manifestaron en el medio PCA (Figura 2e) y en el «Blotter test» (Figura 2f) y cuyos detalles son considerados a continuación:

iii) En el medio PCA la desinfección aplicada a las semillas de ambos cultivares de amaranto produjo incrementos en la germinación de similar magnitud (Tabla 3e).

iv) En el «Blotter test» se registraron leves reducciones, en la germinación en semillas de ambos cultivares pero de similar magnitud (Tabla 3f).

\begin{tabular}{lccc}
\hline $\begin{array}{l}\text { b) medio CYG } \\
\text { Desinfección }\end{array}$ & Cultivar & G erminación & DS \\
\hline 2-CD & 1-Ac & $1,17 \mathrm{a}$ & 0,08 \\
1-SD & 1-Ac & $0,97 \mathrm{a}$ & 0,98 \\
2-CD & 2-A $\mathrm{h}$ & $0,61 \mathrm{~b}$ & 0,62 \\
1-SD & 2-A h & $0,10 \mathrm{c}$ & 0,10 \\
\hline d) medio SA B & & & \\
D esinfección & Cultivar & G erminación & DS \\
\hline 2-CD & 1-AC & $0,35 \mathrm{a}$ & 0,03 \\
2-CD & 2-Ah & $0,10 \mathrm{~b}$ & 0 \\
1-SD & 1-Ac & $0,10 \mathrm{~b}$ & 0 \\
1-SD & 2-Ah & $0,10 \mathrm{~b}$ & 0 \\
\hline f) Blotter test & & & \\
D esinfección & Cultivar & G erminación & D S \\
\hline 1-SD & 1-AC & 1,28 a & 0,09 \\
2-CD & 1-Ac & 1,15 ab & 0,04 \\
1-SD & 2-Ah & 1,02 b & 0,05 \\
2-CD & 2-A h & 1,03 b & 0,03 \\
\hline
\end{tabular}

Análisis complementarios permitieron identificar y comprobar el carácter patógeno de Alternaria alternata (Fr.) Keissler y de Fusarium poae (Peck) Wollenw. A continuación se detallan las características que permitieron su identificación:

-Fusarium poae (Peck) Wollenw: presencia de colonias algodonosas con micelio de color blanquecino; presencia de abundantes microconidios esféricos e hialinos en masas con dimensiones entre 7 a $10 \mu \mathrm{m}$. Macroconidios, escasos, hialinos y con dimensiones que oscilan entre 30-40 x 3-5 $\mu \mathrm{m}$. Escasas Clamidosporas.

-Alternaria alternata (Fr.) Keissler: colonias afelpadas de color verde oliváceo. Conidios de diversas formas: claviformes, piriformes o globosos, muriformes formando largas cadenas. Conidióforos simples o ramificados elevándose en simples o pequeños grupos. Conidios solitarios o en cadenas de 2 a 7. Las dimensiones de los conidios oscilaron entre $15-58 \times 7-11 \mu \mathrm{m}$. 
Interacciones significativas

(a)

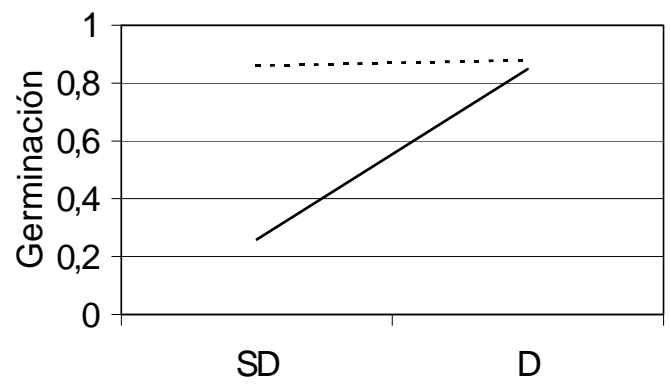

Niveles de desinfección

(c)

APG

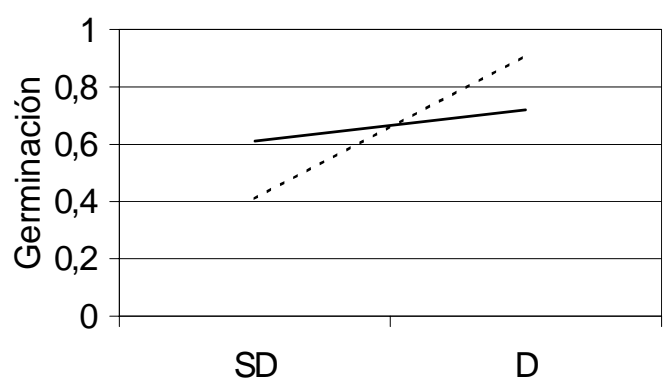

Niveles de desinfección (b)

CYG

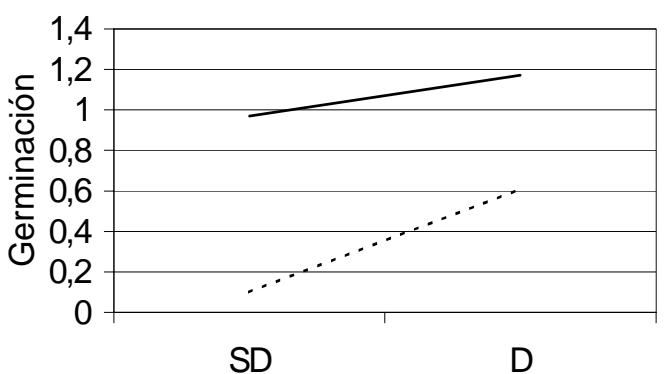

Niveles de desinfección

(d)

$S A B$

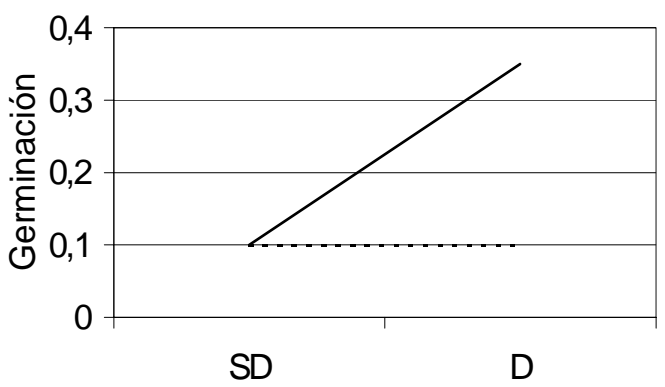

Niveles de desinfección

Interacciones no significativas

(e)

PCA

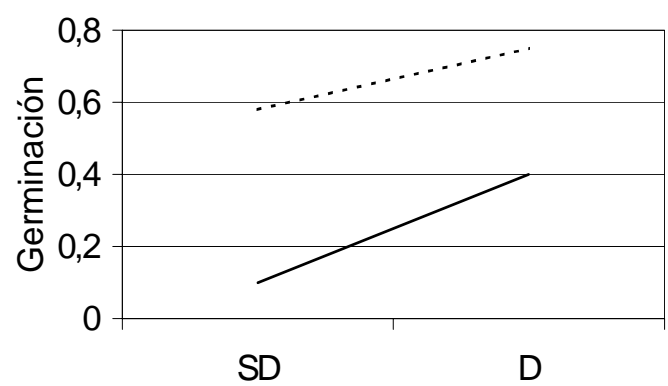

Niveles de desinfección (f)

Blotter test

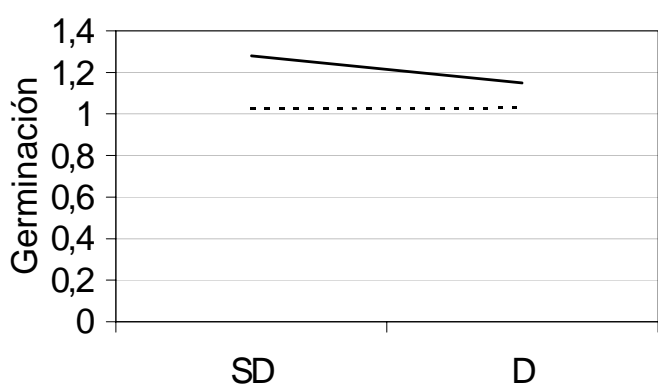

Niveles de desinfección

Figura 2. Evaluación de la germinación de amaranto para cada método de análisis según cultivares de Amaranthus. A. hypochondriacus (línea punteada) y A. cruentus (línea entera). Niveles de desinfección D: con desinfección y SD: sin desinfección. Germinación expresada como el arcoseno de la raíz cuadrada de la proporción de la germinación más 0,01. 
Las semillas inoculadas presentaron la siguiente sintomatología:

-Sistemas radiculares muy pobremente desarrollados, cotiledones unidos, hipoplasia, hipocotiles engrosados (Alternaria alternata)

-Podredumbre de semillas, de raíces, presencia de plántulas marchitas (Fusarium poae).

Las plantas inoculadas con Alternaria alternata desarrollaron lesiones en hojas seguidas por un posterior necrosamiento de los tejidos; en tanto, las plantas que fueron inoculadas con Fusarium poae mostraron un debilitamiento de las mismas seguidas por su posterior marchitamiento.

En infecciones naturales las plantas de ambos cultivares de amaranto desarrolladas a campo presentaron niveles de incidencia para A. alternata responsable del tizón foliar que oscilaron entre un 40 y un $90 \%$ respectivamente para A. cruentus y A. hypochondriacus. En tanto, los niveles de incidencia para Fusarium poae agente responsable de causar el marchitamiento de las plantas fueron del 2 al $4 \%$ para los mismos cultivares analizados.

\section{Discusión}

El número de géneros fúngicos aislados e identificados en las semillas analizadas resultó inesperado; ya que se esperaba encontrar un número menor de contaminantes fúngicos debido al carácter semiárido de la región de procedencia de los cultivares analizados.

La presencia de Alternaria como el género fúngico aislado con mayor frecuencia coincide con lo reportado por Weaver \& Mc Williams, 1980 y Bartolini \& Hampton, 1989. Cabe señalar que dicho género está asociado a enfermedades de pre-emergencia y post-emergencia temprana como el damping off y a enfermedades post-emergentes como el tizón foliar. En el primer caso, Alternaria no actúa solo sino formando un complejo junto con otros géneros fúngicos como Pythium y Fusarium entre otros (Weber, 1987; Cabrera-Nieto, 1989). Los daños causados por el damping off reducen la emergencia de las plantas. En el segundo caso, Alternaria actúa en forma individual afectando al follaje del cultivo provocándole sobre el mismo lesiones que pueden llegar a coalescer en ataques severos (De Sansón, 1989; Stallknecht \& Schulz-Schaeffer, 1993; Sánchez-Enciso et al., 1991).

La elevada frecuencia de aislamiento de $A$. alternata tanto a partir de semillas como del follaje de ambos cultivares de amaranto cultivados a campo sugiere la necesidad de analizar un método de control con la finalidad de reducir la incidencia de dicho patógeno.

El haber utilizado varios métodos de análisis para evaluar la micoflora de semillas de amaranto, confirma lo establecido por Thomson (1983) en cuanto a la cantidad de métodos. Esto se debe a que un solo método de análisis no es suficiente como para permitir el desarrollo de gran parte de la micoflora portada por las semillas.

Tal vez el número de semillas por unidad experimental haya sido elevado, sugiriendo una reducción del mismo para permitir que el trabajo dirigido a la identificación de la micoflora permita combinar representatividad y rapidez en el análisis. Otros autores (Bartolini \& Hampton, 1989) han obtenido resultados (cuatro géneros fúngicos) con un tamaño equivalente a diez semillas de amaranto por caja de Petri; sin embargo, no podemos establecer que exista una relación entre el número de géneros detectados y el tamaño de la muestra, debido a la distinta procedencia geográfica del material: Nueva Zelanda (Bartolini \& Hampton, 1989) y Argentina en el presente trabajo.

De los métodos analizados tres medios agarizados resultaron efectivos en este trabajo, uno de ellos, el APG ya había sido utilizado por Bartolini \& Hampton (1989) para analizar semillas de éste mismo cultivo. Mientras que el empleo de los medios agarizados PCA y $\mathrm{Cz}$ no ha sido reportado en otras investigaciones, aún cuando su uso demostró ser efectivo a nuestros objetivos. 
En cuanto a la desinfección aplicada a las semillas, los resultados muestran coincidencias en general con lo observado por otros autores (Neergard, 1979; Pitt \& Hocking, 1985) principalmente en la implicancia que posee la desinfección en la reducción de hongos de crecimiento expansivo.

En relación a la elevada frecuencia de aislamiento de levaduras en semillas aún no se ha podido determinar cuales han sido los factores que han promovido su desarrollo. Por tal motivo, futuros estudios deberían ser llevados a cabo para estudiar el rol que cumplen las levaduras en las semillas de éste cultivo tan particular.

Con respecto a la evaluación complementaria del poder germinativo la misma nos ha permitido corroborar que el «Blotter test» si bien ha sido el menos efectivo para detectar hongos en semillas, ha sido el más indicado para evaluar la germinación de las semillas de amaranto. Esta última consideración es coincidente con las recomendaciones dadas por el International Seed Testing Association, ISTA (1996) en cuanto a método se refiere. Sin embargo, en este caso en particular no ha sido necesario aplicar ninguno de los tratamientos destinados a romper la dormición de las semillas y que si han sido recomendados por el ISTA (1996) entre las que se incluyen a tratamientos con bajas temperaturas y al uso de nitrato de potasio.

Tanto los métodos de análisis empleados como los niveles de desinfección considerados han afectado al porcentaje de germinación de ambos cultivares de amaranto; los cuales no respondieron en todos los casos en forma similar a pesar de haber sido sometidos a idénticos tratamientos. Esta situación ha impedido que haya sólo una respuesta para explicar los motivos de tales variaciones.

Entre las consideraciones que podemos dar para interpretar por ejemplo los motivos por los cuales las semillas que fueron desinfectadas redujeron su germinación podemos citar:

-La posible desaparición de hongos con ca- pacidad de antagonizar a los patógenos presentes en las semillas.

-El empleo de medios agarizados que ha favorecido más al desarrollo de la micoflora portada por las semillas que a las mismas. Es por ese motivo tal vez que se hayan registrado en general menores valores de germinación en los medios agarizados en relación al «Blotter test», el cual es uno de los métodos más ampliamente utilizados en la valoración de la germinación de las semillas de numerosos cultivos.

Sin embargo el hecho de haber evaluado la germinación en medios agarizados nos ha permitido poder examinar el comportamiento de las semillas bajo condiciones que resultaron desfavorables para las mismas. Dichas condiciones podrían bien simular ciertas situaciones que se producen en el campo y que pueden originar una reducción en la emergencia del cultivo.

\section{Agradecimientos}

Se agradece la colaboración prestada por las Ing. Agrs Mónica Astiz Gazzo y María del Carmen Molina en la revisión del manuscrito.

\section{Literatura Citada}

Afolabi, A.O; O.L. Oke and I.B. Umoh. 1981. Preliminary studies on the nutritive value of some cereal like grains. some cereal like grains. Nutr. Rept Intl 24: 389

Barnett, H.L and B.B. Hunter. 1998. Illustrated genera of Imperfect fungi $4^{\text {th }}$ edition St. Paul. Mn APS Press 218 pp.

Bartolini, J.S and J.G. Hampton. 1989. Grain amaranth. Seed Development, yield and quality in «Proceedings Agronomy Society NZ», 19 pag $55-61$

Becker, R; E.L. Wheeler; K. Lorenz; A.E. Stafford; O.K Grospean; A.A.Betscheart and R.M. Saunders. 1981. A compositional study of amaranth grain. J. Food Sci 46:1175.

Cabrera Nieto, C. 1989. El cultivo de amaranto (Amaranthus spp) una alternativa agronómica para Ecuador. Publicación Miscelánea $\mathrm{N}^{\circ} 52$ Estación Experimental Santa Catalina, Sep. 1989. p. 28

Carlsson, R. 1980. Quantity and quality Amaranths grain from plants in temperate, cold and hot and subtropical climates. A review. In «Proceedings of the Second Amaranth Conference», Rodale 
Press, Emmaus, PA. 48 pp

Covas, G. 1990. Amarantos $i E l$ quinto gran recurso vegetal para la alimentación humana?. Amarantos Novedades e Informaciones $\mathrm{N}^{\circ} 5$, p. 1.

De Sansón, M. P. 1989. Tres enfermedades de los amarantos registrados en la provincia de $\mathrm{La}$ Pampa, República Argentina. Amarantos Novedades e Informaciones $\mathrm{N}^{\circ} 1$ p. 3.

International Seed Testing Association (ISTA). 1996. Seed Science and Technology Rules for Seed Testing Vol 24 Supplements rules 335pp.

National Academy of Sciences. 1975. Underexploited tropical plant with promising economies value. Washington. DC USA. 186pp

Nelson, P.E; T.A. Toussoun and W.F.O. Marasas. 1983. Fusarium species: An Illustrated Manual for Identification. The Pennsylvania State University Press 190pp.

Neergard, P. 1979. Seed pathology. Vol I The Macmillan Press Ltd. 839 pp.

Pitt, J. and Hocking, A. 1985. Fungi and Food spoilage. Academic Press. Sidney, Australia 413 pp.

Sánchez-Enciso, M.C; E. Espitia-Rangel; S. OsadaKawasoe. 1991. Etiología del tizón (Alternaria tenuis) en amaranto (Amaranthus spp.). «Primer Congreso Internacional de amaranto» p. 66.
SAS Institute Inc, SAS/STAT. 1989. User's Guide, Versión 6. Fourth Edition Vol. 2, Cary, NC: SAS Institute Inc. 846pp.

Schmidt, D. 1977. Grain amaranth: A look at some potentials. In: «the Proceedings of the First Amaranth Conference». Rodale Press, Emmaus, PA. 121pp.

Stallknecht, G.F. and J.R. Schulz-Schaeffer. 1993. Amaranth rediscovered. In: J. Janick and J.E. Simon (eds.), New crops. Wiley, New York.p. 211-218

Thomson, J.R. 1983. Introducción a la tecnología de semillas Zaragoza; Acribia 302 pp.

Uzo, J.O and A.U. Okorie. 1983. Amaranthus hybridus. A potential grain crop for West Africa. Nutr. Rep. Intl. 27:519.

Weaver, S.E. and R.L. McWilliams. 1980. The biology of Canadian weeds 44 . Amaranthus retroflexus $\mathrm{L}$ : A. powelli Wats and A. hybridus L. Canadian Journal of Plant Science 60, 1215-1234.

Weber, E. 1987. Amaranth Grain Production Guide 1987 Rodale Research Center Rodale Press. 